|| ISSN(online): 2589-8698 || ISSN(print): 2589-868X ||

International Journal of Medical and Biomedical Studies Available Online at www.ijmbs.info

NLM (National Library of Medicine ID: 101738825)

Index Copernicus Value 2019: 79.34

Original Research Article

Volume 5, Issue 11; November: 2021; Page No. 40-42

\title{
A RARE CASE STUDY OF MEDULLARY CARCINOMA OF DESCENDING COLON REPORTED ON HISTOPATHOLOGY
}

Dr. Rajvala Choudhary ${ }^{1}$, Dr. Chandrika Gupta ${ }^{2}$, Dr. Lakhami Chand Sinsinwar ${ }^{3}$, Dr. Sapna Shrivastava ${ }^{4}$, Dr. Sanjeev Singh Choudhary ${ }^{5}$

${ }^{1}$ Junior Resident, Department of Pathology, RUHS College of Medical Sciences, Jaipur

${ }^{2}$ Associate Professor, Department of Pathology, RUHS College of Medical Sciences, Jaipur

${ }^{3}$ Senior Resident, Department of Surgery, RUHS College of Medical Sciences, Jaipur

${ }^{4}$ Assistant Professor, Department of Pathology, RUHS College of Medical Sciences, Jaipur

${ }^{5}$ Senior Resident, Department of Surgery, SMS Medical College, Jaipur

Article Info: Received 04 October 2021; Accepted 07 November 2021

DOI: https://doi.org/10.32553/ijmbs.v5i11.2285

Corresponding author: Dr. Rajvala Choudhary

Conflict of interest: No conflict of interest.

\section{Abstract}

Medullary carcinoma (MC) of the colon is a rare and unique histologic subtype of colorectal cancer which characterized by poor glandular differentiation and intraepithelial lymphocytic infiltrate. This has now been incorporated as a separate entity in the World Health Organization (WHO) classification of colorectal cancers. It is commonly associated with deficient mismatch repair proteins and has a strong association with Lynch syndrome. Diagnosis is challenging as it does not have the usual immunohistochemical stains on pathology seen in colorectal adenocarcinoma. Here, we discuss an interesting case of $\mathrm{MC}$ of the colon that was metastatic on presentation and constituted a diagnostic challenge ${ }^{1}$.

Keywords: medullary carcinoma, colorectal carcinomas (CRC), medullary carcinoma of colon, poorly differentiated adenocarcinoma, undifferentiated adenocarcinoma.

\section{Introduction}

The incidence of colorectal cancers is increasing throughout the world, the majority of them being adenocarcinoma. Incidence rate of colon cancer is low in India compared to the Western countries. The dietary habits may be the etiological factor. There is significant geographical variation in the incidence rates, and the presentation may also vary ${ }^{1}$.

Medullary carcinoma (MC) of the colon is a rare subtype of colon carcinoma which includes poorly differentiated and undifferentiated histology. The incidence of $\mathrm{MC}$ is estimated to be $0.03 \%$ of all colorectal carcinomas (CRC) $)^{2}$. $\mathrm{MC}$ is twice as prevalent in middle-aged women, favors the right side of the colon, and has a lower risk of lymph node metastasis than colon adenocarcinoma. MC can be poorly differentiated $(72 \%)$ or undifferentiated $(22 \%)$ with solid sheets of cells and excessive intraepithelial lymphocytic infiltration ${ }^{3}$. It does not have the glandular structure seen in adenocarcinoma ${ }^{3}$.

\section{Case presentation}

A 67-year-old female presented with the chief complaints of right side abdominal pain, abdominal distension, and constipation for 6 months. She also presented with history of nausea, vomiting, weight loss and fatigue over 6 months period of time. As the patient's symptoms worsened, she was referred to the surgical outpatient clinic of our hospital.

Laboratory evaluation was unremarkable, including complete blood count and biochemistry panel. The laboratory data showed the patient had a white blood cell count of $6940 / \mathrm{mm} 3$, a hemoglobin level of $5.3 \mathrm{~g} / \mathrm{dL}$, a hematocrit level of $18.1 \%$, and a platelet count of $2,96,000 / \mathrm{mm} 3$. The patient's electrolyte and blood urea levels were normal, as was her liver function.

An abdominal X-ray examination revealed a dilated colon. These findings were consistent with bowel obstruction. Ultrasound showed a well defined irregular solid mass seen with central cystic areas, approx. size of mass was $55 \mathrm{x}$ $73 \mathrm{~mm}$ seen in left iliac fossa and extending into left lumbar area. Mass lied adjacent to bowel loop. Solid area showed vascularity. Ultrasound was suggestive of possibility of GIST. A contrast enhanced computed tomography (CECT) of the abdomen revealed an ill-defined large exophytic mass lesion approx. $68 \times 65 \times 75 \mathrm{~mm}$ seen in left lumbar region and left iliac fossa region arising from descending colon. There was fistulous communication of mass lesion with descending colon with presence of oral contrast within the centre of mass. Contrast enhanced computed 
tomography (CECT) findings were suggestive of a large obstructive exophytic mass lesion arising from descending colon with fistulous communication with colonic lumenlikely neoplastic etiology (?GIST), responsible for distension of the right colon. The patient underwent exploratory laparotomy then lump excision with bowel resection and anastomosis.
The biopsy specimen included the partial colectomy $(35 \mathrm{~cm}$ in length $\times 6.0 \mathrm{~cm}$ in internal circumference) witha $8 \mathrm{~cm} \mathrm{x}$ $7.0 \mathrm{~cm}$ centrally ulcerated, friable, fungating, circumferential mass of colon, which was widely clear of the proximal and distal margins. The mesenteric margins appear uninvolved by the tumor, but focally involved the subserosal adipose tissue.

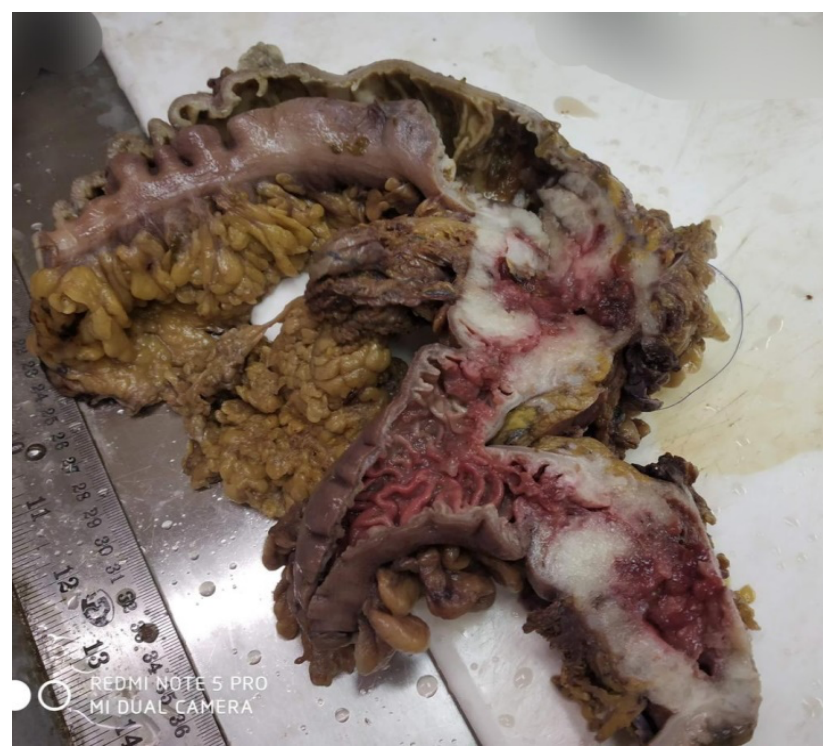

\section{Gross Picture of Specimen}

A histopathological examination with hematoxylin and eosin staining of the resected lesion revealed medullary type poorly differentiated adenocarcinoma. Histology of the lesion showed solid nests of tumor cells arranged in lobules. Cells are round to oval having abundant vacuolated cytoplasm with round nuclei and prominent nucleoli. Signet ring cells are also seen. At few foci the tumor cells are forming glands and singly scattered in mucin pools. The tumor showed infiltrating up to serosa. The surrounding tissue show lymphocytic infiltration. No metastatic regional lymph nodes were identified and both resected margins were free from tumor. The patient had an uneventful recovery and was discharged from the hospital on the 10th day after surgery.

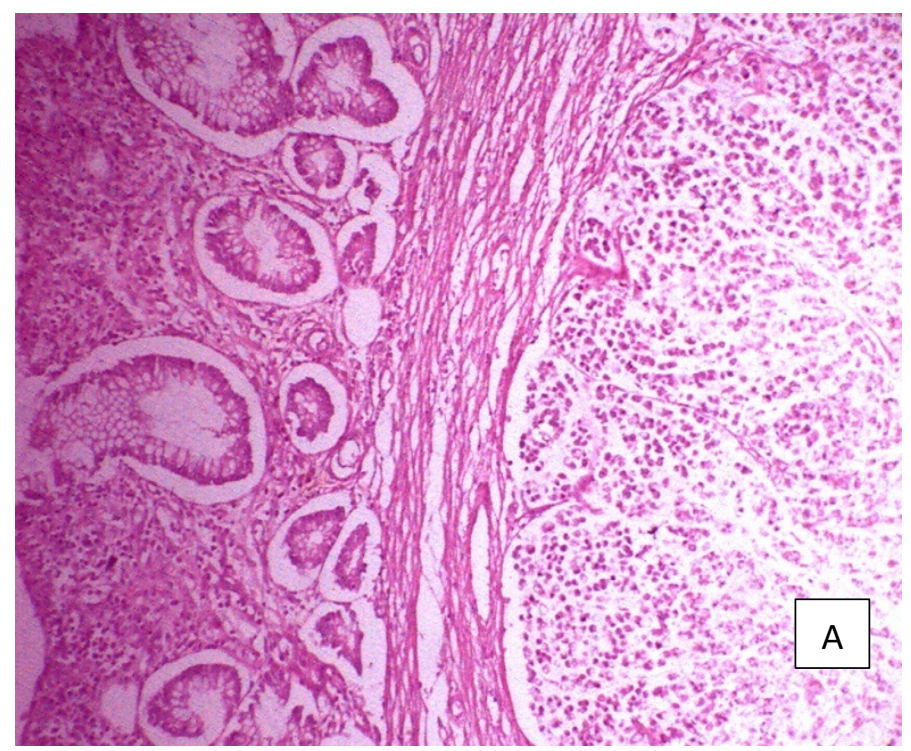




\begin{tabular}{|l|r|}
\hline Rajvala Choudhary et al. & International Journal of Medical and Biomedical Studies (IJMBS) \\
\hline
\end{tabular}

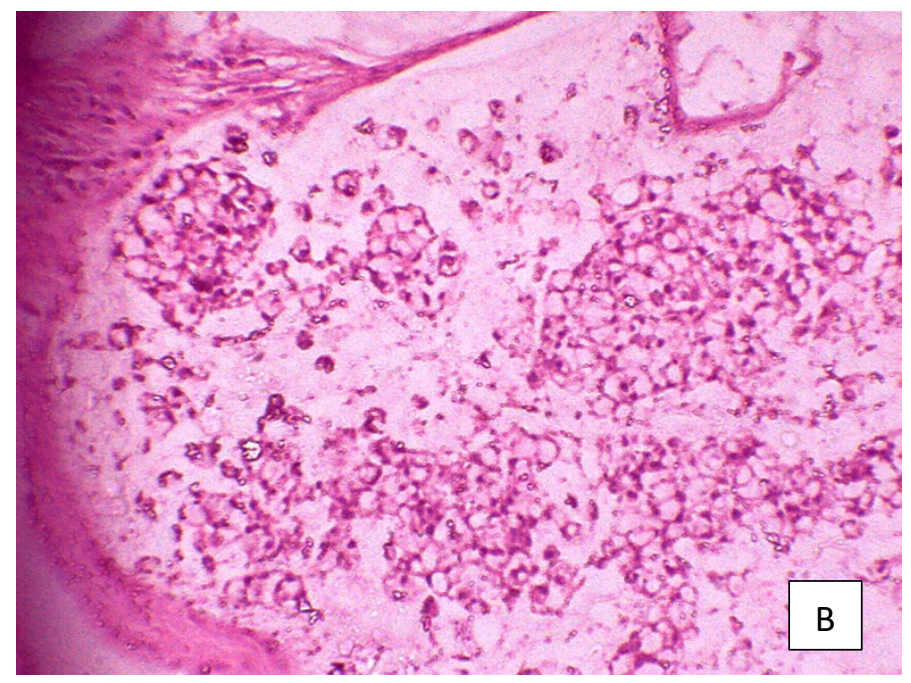

H\&E stain of the biopsy at low magnification reveals solid nests of tumor cells arranged in lobules with abundant intratumoral lymphocytes (A). Higher magnification reveals moderately to severely pleomorphic cells with vacuolated cytoplasm, round nuclei and prominent nucleoli (B).

\section{Conclusions}

$\mathrm{MC}$ of the colon is a distinct but extremely rare variant of adenocarcinoma with unique clinical, histological, immunophenotypical, and prognostic characteristics. In conclusion, $\mathrm{MC}$ of the colon differs from the adenocarcinoma in terms of clinical and immunohistochemical characteristics. Due to its atypical histopathological presentation, immunohistochemical staining and morphological diagnosis are needed for accurate diagnosis. Medullary variant of poorly differentiated adenocarcinoma has a better prognosis than other variants of poorly differentiated adenocarcinoma ${ }^{4}$.

\section{References}

1. Medullary carcinoma in the colorectum: a systematic review and meta-analysis. Pyo JS, Sohn JH, Kang G. Hum Pathol. 2016;53:91-96. [PubMed] [Google Scholar]

2. Colorectal Cancer in India: An Audit from a Tertiary Center in a Low Prevalence Area Indian J SurgOncol. 2017 Dec; 8(4): 484-490.

3. Fatima, Zainab et al. "Medullary Carcinoma of the Colon: A Histopathologic Challenge." Cureus vol. 13,6 e15831. 22 Jun. 2021, doi:10.7759/cureus. 15831

4. Medullary carcinoma of the large intestine: a population based analysis.[Int J Oncol. 2010]

5. Medullary adenocarcinoma of the colon: clinicopathologic study of 11 cases.[Hum Pathol. 1999] 